\title{
Tumor budding outperforms ypT and ypN classification in predicting outcome of rectal cancer after neoadjuvant chemoradiotherapy
}

\author{
Iryna Trotsyuk ${ }^{1}$, Halina Sparschuh ${ }^{1}$, Alice Josephine Müller ${ }^{1}$, Konrad Neumann $^{2,3}$, Martin Kruschewski ${ }^{4}$, \\ David Horst ${ }^{1}$ and Sefer Elezkurtaj ${ }^{1 *}$
}

\begin{abstract}
Background: Budding is a complementary prognostic factor for colorectal cancer. In this study, we aimed to clarify the role of tumor budding in rectal cancer patients after preoperative chemoradiotherapy.

Methods: A total of 124 patients with rectal cancer treated with neoadjuvant chemoradiotherapy and consecutive surgery were included. Surgical specimens were evaluated for budding and routine clinicopathological features. Budding was evaluated on hematoxylin and eosin (H\&E)-stained slides and by cytokeratin immunohistochemical (IHC) staining.

Results: A budding rate of 36.9\% $(n=38)$ by H\&E and 55.6\% $(n=55)$ by $\mathrm{HC}$ was observed. Budding was significantly associated with a high ypT and ypN status, poor differentiation, and low degrees of tumor regression. Moreover, budding was strongly predictive of a worse patient outcome, as measured by tumor recurrence or death. In multivariate analyses, budding remained the only significant parameter for overall survival and was even superior to the ypT and ypN status (budding in H\&E: hazard ratio (HR) 2.72, 95\% confidence interval (95\% Cl) 1.15-6.44, $p=$ 0.023; budding in IHC: HR 5.19, 95\% Cl 1.62-16.61, $p=0.006$ ).

Conclusion: Budding is a strong prognostic predictor of survival in rectal cancer patients after neoadjuvant therapy. A standardized evaluation of tumor budding after neoadjuvant therapy may thus aid in risk stratification and guide the clinical management of patients with rectal cancer. Immunostaining can help to enhance the diagnostic accuracy and prognostic significance.
\end{abstract}

Keywords: Rectal cancer, Neoadjuvant therapy, Tumor budding, Prognostic factor

\section{Background}

Locally advanced rectal cancers are treated with preoperative local radiation and simultaneous chemotherapy. Since the implementation of this therapy, the risk for local recurrence has notably decreased, and sphincter-preserving surgery is more often performed [1, 2]. After such intensive therapy, the initial morphology of the tumor is subject to considerable changes. Nevertheless, the evaluation of these histologically changed

\footnotetext{
*Correspondence: sefer.elezkurtaj@charite.de

${ }^{1}$ Institut für Pathologie, Charité - Universitätsmedizin Berlin, Campus Charité Mitte, Charitéplatz 1, 10117 Berlin, Germany

Full list of author information is available at the end of the article
}

cancers remains the same as for tumors without neoadjuvant therapy. The tumor, node, metastasis (TNM) staging system, which is continuously updated, is widely used to predict outcomes and aids in clinical decision making in colorectal cancer. However, after preoperative therapy, the prognostic impact of the classical TNM system is subject to certain limitations, especially in tumors with wide fibrotic areas, i.e., a major therapeutic response [3]. Furthermore, the assessment of tumor regression, the relation of residual cancer cells to fibrosis, is an important feature of pathohistological evaluation protocols of rectal cancer after neoadjuvant therapy. Other morphological parameters have not yet been

(c) The Author(s). 2019 Open Access This article is distributed under the terms of the Creative Commons Attribution 4.0 International License (http://creativecommons.org/licenses/by/4.0/), which permits unrestricted use, distribution, and 
clarified as to whether they play a decisive role in the prognostic prediction of treated rectal cancer. They might constitute, however, a valuable addition to the TNM system and regression grading of treated rectal cancers.

An example of such a morphological parameter, the role of which is not yet clear in tumors treated with neoadjuvant therapy, is tumor budding. In rectal cancers without preoperative multimodality treatment, budding is associated with lymphovascular invasion [4-6], metastatic lymph nodes $[4,6-8]$, a higher TNM stage $[6,8]$, and distant metastasis [6,9]. In colorectal carcinoma, budding is a strong adverse prognostic marker [4-9].

The aim of this retrospective study was to investigate whether tumor budding is a prognostic factor for survival in patients with rectal cancer who received neoadjuvant chemoradiotherapy. Special attention was paid to tumor budding assessed with the method introduced by Ueno et al. [10].

\section{Methods \\ Patients}

The study cohort included 124 consecutive patients with a biopsy-proven diagnosis of rectal adenocarcinoma and received radical surgery after neoadjuvant treatment between 2002 and 2011. All patients provided written consent to further investigate their tissue samples as well as the anonymous use of their clinical data. Patients under the age of 18 were not included in the study. Investigations on archived tissue and anonymized data were approved by the institutional ethics board (№. EA1/370/ 16). Primary clinical parameters and survival data were obtained from electronic health records. Distant metastasis had been excluded or detected at diagnosis and during the follow-up by abdominal ultrasound and chest radiography according to national guidelines. In cases of suspicion or ambiguity, computed tomography (CT) scans were performed [11]. Missing survival data at follow-up were gathered by delivering a questionnaire to the primary care physicians of the patients.

\section{Neoadjuvant therapy and surgery}

All 124 included patients received long-course neoadjuvant therapy. Eighty patients were treated in strict compliance with the standard regimen, defined by a cumulated radiation dose of 50.4 Gy applied in 5 weekly fractions of $1.8 \mathrm{~Gy}$ using $18 \mathrm{MeV}$ photons. These patients received a continuous infusion of $225 \mathrm{mg} 5$-FU per day and square meter of body surface for the duration of radiotherapy. Most of the remaining 44 patients received only slightly variant chemotherapy along with hyperfractionated radiation. After an interval of 4-6 weeks, total mesorectal (TME) surgery was performed.

\section{Pathological assessment}

The quality of the total mesorectal excision was assessed using the Quirke criteria. Quirke grade 1 (poor) corresponds to irregular mesorectal fascia, with defects or incisions up to 1 square $\mathrm{cm}$ to the muscularis propria, irregular circumferential resection margin with small amount of mesorectal fat and low anterior safety margin. Quirke grade 2 (suboptimal) means that there is a moderate amount of mesorectum with some irregularity; moderate distal coning may be present. Quirke grade 3 (optimal) indicates that there is a good amount of mesorectum, a smooth surface, a good safety distance at the frontside and no defects in the mesorectum. Tissue sections were prepared from paraffin-embedded samples, mounted onto glass slides, stained with hematoxylineosin according to standard procedures, and examined with a Nikon ECLIPSE E200 microscope and a $\times 10 \mathrm{ocu}-$ lar lens. The pathological $\mathrm{T}$ and $\mathrm{N}$ stage (ypT and ypN, respectively) were evaluated according to the 7th AJCC TNM classification. The tumor regression grade of the resected tumor was assessed using the original score proposed by Dworak et al. [12]. Tumor regression was described as follows: Grade 0: no regression; Grade 1: dominant tumor mass with obvious fibrosis and/or vasculopathy; Grade 2: dominant fibrotic changes with few tumor cells or groups (easy to find); Grade 3: very few (difficult to find microscopically) tumor cells in fibrotic tissue; and Grade 4: no tumor cells, only a fibrotic mass (total regression or response). All formerly determined histopathological features, such as regression grade or pathological $\mathrm{T}$ and $\mathrm{N}$ stage, were retrospectively reevaluated by one trained observer (I.T.) who was blinded to patient outcomes and reviewed by a specialist gastrointestinal pathologist (S.E.).

\section{Tumor budding}

Tumor budding was defined as a single tumor cell or a cluster of up to four tumor cells in the invasive front of the tumor or within the tumor. For quantifications, the sections were first scanned at a low power, and an area with maximal budding was identified. Then, tumor buds were counted in one field measuring $0.785 \mathrm{~mm}^{2}$ using a $20 x$ objective lens. A field with five or more buds was viewed as budding positive (BD-1), while a field with four or fewer buds was viewed as budding negative (BD0) [10].

\section{Immunohistochemical staining}

In addition, to better understand tumor budding after neoadjuvant treatment and to highlight buds in detail, we also performed immunohistochemical (IHC) staining. There were 99 corresponding unstained tissue slides available for immunohistochemistry with a pancytokeratin antibody AE1/AE3. Staining was performed 
according to standard protocols provided by the automated Ventana BenchMark XT immunostainer (Ventana Medical Systems, Inc., Tucson, AZ, USA). Briefly, the tissue sections were deparaffinized and rehydrated and subjected to heat-induced epitope retrieval and endogenous peroxidase blocking with $\mathrm{H}_{2} \mathrm{O}_{2}$. Subsequently, the slides were incubated with a primary pan-cytokeratin antibody (clone AE1/AE3, dilution 1:500, DAKO) for 60 min and then with a horseradish peroxidase (HRP)-conjugated secondary antibody (DISCOVERY Universal Secondary Antibody (RUO)) for $32 \mathrm{~min}$. This was followed by applying the chromogen $3,3^{\prime}$-diaminobenzidine-tetrahydrochloride (DAB) for $8 \mathrm{~min}$ and counterstaining with hematoxylin and bluing reagent (Ventana Medical Systems, Inc.) for $12 \mathrm{~min}$. Budding was evaluated on IHCstained slides with the same method and cut-off as described above for H\&E-stained slides.

\section{Statistical analysis}

We compared the BD-0 and BD-1 groups using the chisquared test or Fisher's exact test, as appropriate. Comparisons of the means of metrical variables, such as age, BMI, tumor size, circumferential resection margin between the BD-0 and BD-1 groups were performed using a t-test for independent samples. Univariate and multivariate Cox proportional hazards regression models were used to estimate hazard ratios (HRs) with 95\% confidence intervals (95\% CIs). For categorical variables, the lowest value served as the reference category. The categorical variables used in the univariate analyses were gender (male versus female), American Society of Anesthesiologists (ASA) classification (2 and 3 versus 1 ), cM stage, type of resection (Abdominoperineal resection versus lower anterior resection), adjuvant therapy, Quirke Grade (poor versus moderate and good), higher ypT stage (ypT3-4 versus ypT0-2), positive ypN stage (ypN+ versus ypN0), higher histological and regressive grading, vascular and perineural invasion (V1, L1, Pn1), quality of resection ( $\mathrm{R}+$ versus R0) and positive budding. The continuous variables used in univariate analyses were age at surgery, body mass index (BMI), tumor size and circumferential resection margin (CRM). Covariates and factors included in the multivariate regression analysis were budding and ypT and ypN stage. Dichotomization of ypT and ypN stages was used to avoid overfitting of the model. The primary endpoints of the study were the hazard ratios for diseasefree survival (DFS) and overall survival (OS). Overall survival (OS) was defined as the time from the date of surgery to the date of death from any cause. Disease-free survival (DFS) was defined as the time from the date of surgery to the date of pelvic recurrence and/or distant disease or death from any cause. Kaplan-Meier survival curves show the influence of tumor budding on survival. The different budding categories (BD-0 and BD-1) in the plotted DFS and OS curves were compared using the log-rank test. With the kappa value, we evaluated the consistency of H\&E-stained slides with IHC-stained slides. All tests were two-sided, and the level of significance was set at $\alpha=0.05$. We performed all statistical analyses using SPSS Statistics 24.0 Software (SPSS, Inc., Chicago, IL).

\section{Results}

The initial study cohort included 124 patients, 87 men (70.2\%) and 37 women (29.8\%), with a mean age of 64.7 years (range 34-87 years). The TNM classification before neoadjuvant treatment was composed as follows: cT2 in 12 patients $(9.7 \%)$, cT3 in 92 patients $(74.2 \%)$ and cT4 in 20 patients (16.1\%); cN0 in 14 patients $(11.3 \%)$ and $\mathrm{cN}+$ in 110 patients (88.7\%); cM0 in 111 (89.5\%) and cM1 in $13(10.5 \%)$ patients. Lower anterior resection (LAR) was performed on 92 patients (74.2\%), abdominal perineal resection on 32 patients (25.8\%). The average number of harvested lymph nodes was 18 (range 5-67). The quality of mesorectum specimens was poor in $5(4.0 \%)$, suboptimal in $19(15.3 \%)$ and optimal in $86(69.4 \%)$ resected rectal cancers. In $14(11.3 \%)$ it was not described. A complete resection with R0 status was achieved in 118 patients (95.2\%). Adjuvant chemotherapy was received in $71(57.3 \%)$ cases, while in $32(25.8 \%)$ cases the tumor board decided against adjuvant therapy. For 21 (16.9\%) patients information on adjuvant therapy was not available.

There were seven local recurrences (5.6\%) and eighteen distant recurrences (14.5\%) in the follow-up period. Thirty-two patients $(25.8 \%)$ died during the follow-up period. The mean time for recurrence was 28.4 months (standard deviation 24.6 months, maximum 94 months). The mean follow-up time was 54.7 months (standard deviation 35.5 months, maximum 134 months).

\section{Evaluation of tumor budding by H\&E and IHC}

In the following tumor budding analyses, we examined only specimens with residual tumor. Twenty-one specimens with pathological complete response (pCR) were excluded from further statistical analyses. Without 21 pCR cases, there were 103 cases available for analysis of H\&E-stained tissue sections, and 99 cases for analysis of IHC-stained tissue sections (Fig. 1). The examination of H\&E-stained sections showed 38 (36.9\%) buddingpositive cases and $65(63.1 \%)$ budding-negative cases. On IHC-stained sections, 44 cases $(44.4 \%)$ were budding negative, and 55 cases (55.6\%) were budding positive; thus, there was a higher percentage compared to H\&Estained slides. Figure 2 illustrates an example of a budding-negative case and a budding-positive case from both staining methods.

To evaluate the consistency of budding evaluated on H\&E- or IHC-stained sections, we used fourfold tables 


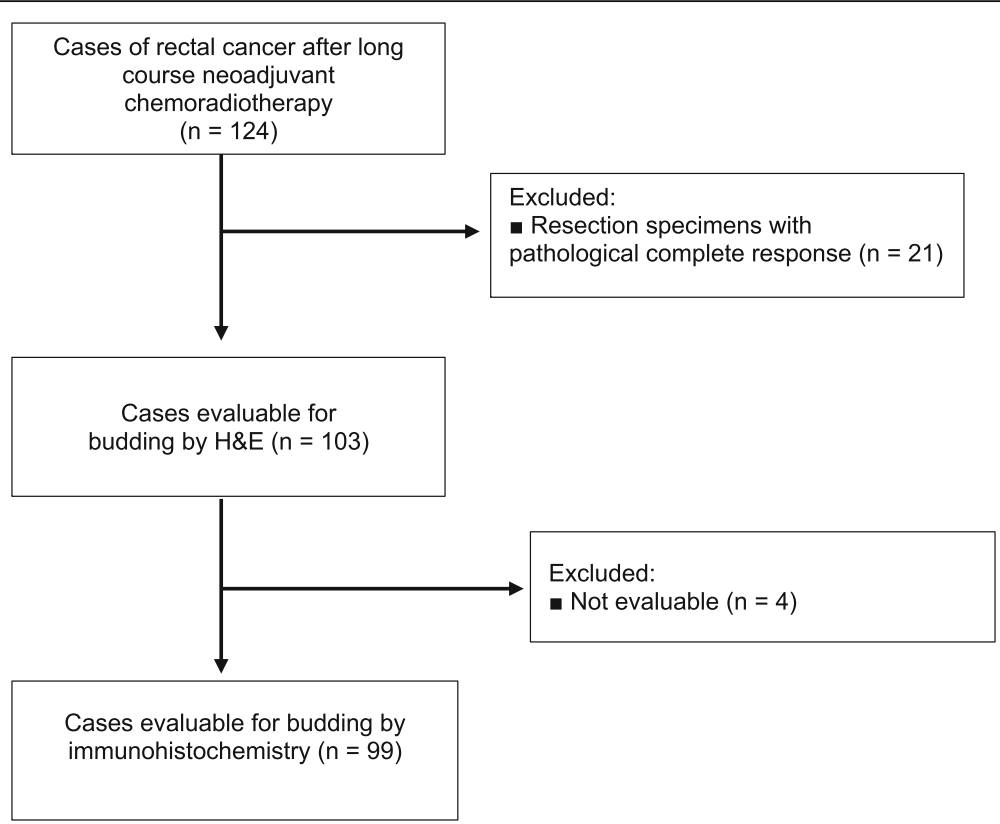

Fig. 1 Flow chart of histological analysis for the study cohort

(Table 1). After immunohistochemical staining, there were more budding-positive cases. The assessment of tumor budding on H\&E and IHC reached good agreement, with a kappa value of 0.609 . The evaluation of budding on H\&E-stained slides compared well with the evaluation of budding on IHC-stained slides.

\section{Tumor budding is associated with adverse clinicopathological features}

As analyzed by H\&E staining, budding was found more frequently in specimens of elderly patients $(p=0.032)$, in patients with a lower BMI $(p=0.042)$ and in patients with a higher ASA score $(p=0.022)$. Patients in whom
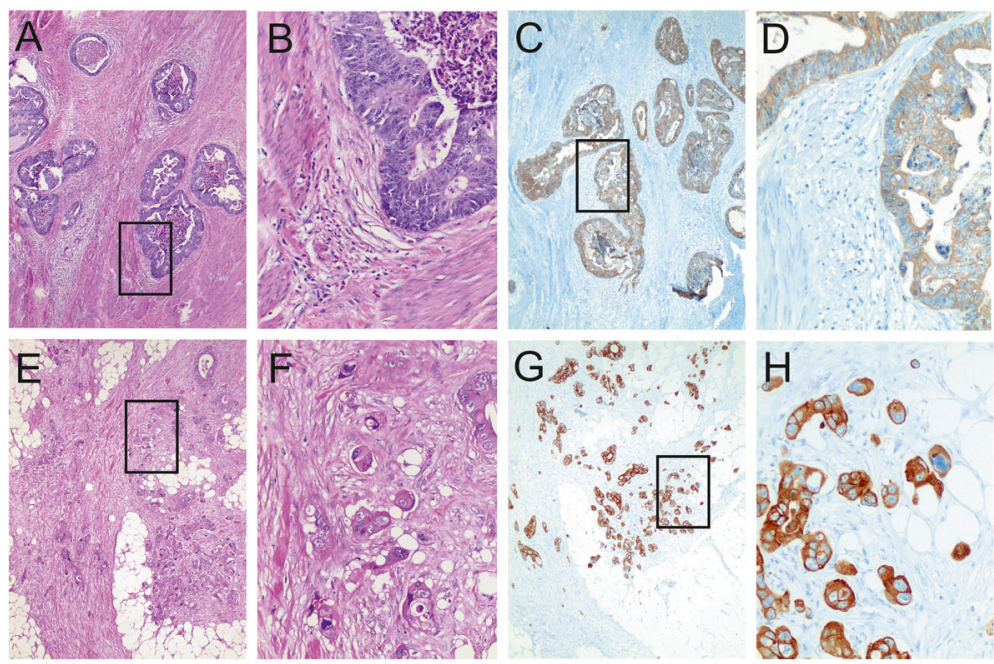

Fig. 2 Comparison of tumor budding in neoadjuvant treated rectal cancers in both hematoxylin-eosin and in immunohistochemical staining. Tumor budding was defined as a single tumor cell or a cluster up to four tumor cells at the invasive front or within the tumor as well. Tumor buds were counted in one field measuring $0.785 \mathrm{~mm}^{2}$ using a 20x objective lens. A field with 4 buds or fewer was viewed as budding negative (a-d), a field with 5 or more buds was viewed as budding positive (e-h). Boxed areas are shown in a higher magnification on the right side of the corresponding picture. (Original magnification and staining method: (a) $\times 40, \mathrm{H \& E} ;(\mathbf{b}) \times 200, \mathrm{H \& E} ;(\mathbf{c}) \times 40, \mathrm{AE} 1 / \mathrm{AE} 3$; (d) $\times 200, \mathrm{AE} 1 / \mathrm{AE} 3$; (e) $\times 40$, $\left.\mathrm{H} \& \mathrm{E}_{;}(\mathbf{f}) \times 200, \mathrm{H} \& \mathrm{E}_{;}(\mathbf{g}) \times 40, \mathrm{AE} 1 / \mathrm{AE} 3 ;(\mathbf{h}) \times 200, \mathrm{AE} 1 / \mathrm{AE} 3 ;\right)$ 
Table 1 Comparison of cases where budding was evaluated on H\&E with cases budding evaluated on IHC

\begin{tabular}{lllll}
\hline & & \multicolumn{2}{l}{ Budding on $\mathrm{HC}$} & \\
\cline { 3 - 4 } & & negative & positive & $\mathrm{n}$ \\
\hline Budding on H\&E & negative & 44 & 20 & 64 \\
& positive & 0 & 35 & 35 \\
& $\mathbf{n}$ & 44 & 55 & 99 \\
\hline
\end{tabular}

lower anterior resection was performed had significantly less budding $(p=0.004)$. Budding was also significantly associated with a higher $\mathrm{T}$ stage, both before and after neoadjuvant therapy (for cT stage $p=0.005$ and for ypT stage $p=0.001$ ), metastatic lymph nodes (for ypN stage $p=0.006$ ), a poorer level of histological differentiation $(p=0.021)$, a lower response according to Dworak's tumor regression grading scale $(p=0.001)$, venous or perineural invasion ( $p=0.030$ and $p=0.001$ ), and a worse outcome in subsequent observations, such as cancer recurrence $(p=0.002)$ or death $(p=0.006)$. Tumors with a smaller circumferential resection margin and a poor quality of mesorectal excision (Quirke Grade poor) also had significantly more budding $(p=0.010$ and $p=$ 0.004 , respectively). There were no significant associations between budding and sex $(p=0.501)$, the mean macroscopically evaluated tumor size $(p=0.192)$, synchronous metastasis at the time of surgery $(p=0.066)$, or metastatic lymph nodes before neoadjuvant treatment $(p=0.742)$. The parameters mentioned above can be found in Tables 2 and 3. The clinicopathological features of budding in IHC-stained specimens are listed in Tables 2 and 3.

\section{Many factors influence the survival of rectal cancer patients}

In univariate analysis, factors such as a higher ypT stage, metastatic lymph nodes, vascular and perineural invasion, synchronous metastasis at the time of surgery, poorly differentiated and macroscopically larger tumors and positive budding (evaluated in both staining methods) had a significantly negative impact on diseasefree survival. A higher BMI, larger circumferential resection margins on tumor specimens and more regression had a significantly positive impact on disease-free survival. All these variables with the corresponding hazard ratios can be found in Table 4 .

In univariate analyses on overall survival, age and synchronous metastasis at the time of surgery, a poorer quality mesorectal excision, a higher yp $\mathrm{T}$ stage, perineural invasion, poorly differentiated tumors and positive budding, independent of the staining method, had a significantly negative impact on overall survival. Lymph node stage had no significant influence on overall survival. Similar to disease-free survival, prolonged overall survival was associated with tumor specimens with larger circumferential resection margins and patients with more regression and in patients with a higher BMI. All these variables with the corresponding hazard ratios can be found in Table 5 .

Budding is an independent prognostic factor for diseasefree survival and overall survival in multivariate cox proportional hazards regression models

In the multivariate analysis, budding scored on H\&Estained sections (HR 2.34, 95\% CI 1.14-4.79; $p=0.020$ ) and ypT stage (HR 2.85, 95\% CI 1.16-7.02; p = 0.023) were both independent predictors of disease-free survival (Table 6). In the multivariate analysis, when budding was evaluated on IHC-stained sections, positive budding (HR 4.59, 95\% CI 1.79-11.72; p = 0.001) remained the only independent prognostic factor (Table 7). In the multivariate regression analysis of overall survival, only tumor budding remained a significant parameter (H\&E: HR 2.72, 95\% CI 1.15-6.44, $p=0.023$; IHC: HR 5.19, $95 \%$ CI 1.62-16.61, $\mathrm{p}=0.006)$ and was even superior to the ypT and ypN status (Tables 8 and 9).

\section{The prognostic impact of budding is confirmed by Kaplan-Meier survival analysis}

With the H\&E staining method, for patients with budding-positive tumors, the five-year disease-free survival rate was $39.0 \%$, and for those without budding, the rate was $75.0 \%$. With the IHC staining method, for patients with budding-positive tumors, the five-year disease-free survival rate was $44.0 \%$, and for those without budding, the rate was $87.0 \%$. Furthermore, for patients with positive budding evaluated on H\&E-stained sections, the five-year overall survival rate was $53.0 \%$, and for those without budding, the rate was $84.0 \%$. On IHC-stained sections, the five-year overall survival rate was $59.0 \%$ for patients with budding-positive tumors and $92.0 \%$ for those without budding. Independent of the staining method, patients with positive budding had significantly poorer DFS and OS compared to those without budding (Fig. 3).

\section{Discussion}

In the present study, we investigated whether tumor budding is a prognostic factor in patients with rectal adenocarcinoma treated with neoadjuvant therapy. Our results showed a strong connection between posttreatment budding and a more aggressive tumor biology, i.e., correlation with adverse clinicopathological features, such as deeper tumor infiltration or a higher frequency of lymph node metastases. Irrespective of the staining method used, patients with tumor budding had a significantly worse prognosis for disease-free survival and overall survival. These aspects have already been 
Table 2 Tumor budding and associations with clinical features

\begin{tabular}{|c|c|c|c|c|c|c|c|c|c|c|}
\hline \multirow{3}{*}{ All patients, n (\%) } & \multicolumn{5}{|c|}{ Budding on H\&E } & \multicolumn{5}{|c|}{ Budding on IHC } \\
\hline & \multicolumn{2}{|c|}{ negative } & \multicolumn{2}{|c|}{ positive } & \multirow[t]{2}{*}{$P$} & \multicolumn{2}{|c|}{ negative } & \multicolumn{2}{|c|}{ positive } & \multirow[t]{2}{*}{$P$} \\
\hline & 65 & (63.1) & 38 & $(36.9)$ & & 44 & $(44.4)$ & 55 & (55.6) & \\
\hline \multicolumn{11}{|l|}{ Age } \\
\hline Mean, years (SD) & 62.5 & $(9.2)$ & 67.1 & (10.8) & 0.032 & 61.8 & (9.8) & 65.7 & (9.8) & 0.055 \\
\hline \multicolumn{11}{|l|}{ Sex, n (\%) } \\
\hline Male & 48 & $(65.8)$ & 25 & $(34.2)$ & 0.501 & 34 & $(47.9)$ & 37 & (52.1) & 0.370 \\
\hline Female & 17 & $(56.7)$ & 13 & $(43.3)$ & & 10 & (35.7) & 18 & $(64.3)$ & \\
\hline \multicolumn{11}{|l|}{ BMI } \\
\hline Mean, $\mathrm{kg} / \mathrm{m}^{2}$ (SD) & 25.8 & (3.9) & 23.6 & $(5.7)$ & 0.042 & 25.6 & $(3.2)$ & 24.6 & (5.6) & 0.252 \\
\hline \multicolumn{11}{|c|}{ ASA classification, n (\%) } \\
\hline 1 & 12 & $(85.7)$ & 2 & $(14.3)$ & 0.022 & 9 & $(64.3)$ & 5 & (35.7) & 0.096 \\
\hline 2 & 47 & $(64.4)$ & 26 & (35.6) & & 31 & $(44.9)$ & 38 & (55.1) & \\
\hline 3 & 6 & $(37.5)$ & 10 & $(62.5)$ & & 4 & (25.0) & 12 & (75.0) & \\
\hline \multicolumn{11}{|l|}{ CT stage, n (\%) } \\
\hline T0-1 & - & - & - & - & 0.005 & - & - & - & - & 0.011 \\
\hline $\mathrm{T} 2$ & 5 & (55.6) & 4 & $(44.4)$ & & 2 & (22.2) & 7 & (77.8) & \\
\hline T3 & 54 & (72.0) & 21 & $(28.0)$ & & 39 & (53.4) & 34 & $(46.6)$ & \\
\hline $\mathrm{T} 4$ & 6 & (31.6) & 13 & $(68.4)$ & & 3 & (17.6) & 14 & (82.4) & \\
\hline \multicolumn{11}{|l|}{ cN stage, n (\%) } \\
\hline NO & 7 & $(70.0)$ & 3 & $(30.0)$ & 0.742 & 7 & (70.0) & 3 & $(30.0)$ & 0.104 \\
\hline $\mathrm{N}+$ & 58 & (62.4) & 35 & $(37.6)$ & & 37 & (41.6) & 52 & (58.4) & \\
\hline \multicolumn{11}{|l|}{ cM stage, n (\%) } \\
\hline MO & 60 & $(66.7)$ & 30 & (33.3) & 0.066 & 42 & $(47.2)$ & 47 & (52.8) & 0.178 \\
\hline M1 & 5 & (38.5) & 8 & (61.5) & & 2 & (20.0) & 8 & $(80.0)$ & \\
\hline \multicolumn{11}{|c|}{ Type of resection, n (\%) } \\
\hline LAR & 53 & (71.6) & 21 & $(28.4)$ & 0.004 & 39 & (53.4) & 34 & (46.6) & 0.003 \\
\hline APR & 12 & $(41.4)$ & 17 & (58.6) & & 5 & $(19.2)$ & 21 & $(80.8)$ & \\
\hline \multicolumn{11}{|c|}{ Adjuvant therapy, n (\%) } \\
\hline Yes & 42 & $(66.7)$ & 21 & (33.3) & 0.218 & 27 & $(45.0)$ & 33 & $(55.0)$ & 0.741 \\
\hline No & 12 & $(52.2)$ & 11 & $(47.8)$ & & 9 & $(40.9)$ & 13 & $(59.1)$ & \\
\hline Not described & 11 & & 6 & & & 8 & & 9 & & \\
\hline \multicolumn{11}{|c|}{ Relapse in the follow-up period, $\mathrm{n}(\%)$} \\
\hline No & 57 & $(71.3)$ & 23 & $(28.7)$ & 0.002 & 41 & (53.9) & 35 & $(46.1)$ & 0.001 \\
\hline Yes & 8 & $(34.8)$ & 15 & $(65.2)$ & & 3 & (13.0) & 20 & $(87.0)$ & \\
\hline \multicolumn{11}{|l|}{ Type of relapse, n (\%) } \\
\hline Local recurrence & 2 & $(50.0)$ & 2 & $(50.0)$ & 0.015 & 1 & (25.0) & 3 & $(75.0)$ & 0.003 \\
\hline Liver & 2 & (28.6) & 5 & (71.4) & & 0 & (0) & 7 & $(100)$ & \\
\hline Lung & 3 & (33.3) & 6 & $(66.7)$ & & 1 & $(11.1)$ & 8 & $(88.9)$ & \\
\hline Cerebral & 1 & (33.3) & 2 & $(66.7)$ & & 1 & (33.3) & 2 & $(66.7)$ & \\
\hline \multicolumn{11}{|c|}{ Survival status in follow-up, n (\%) } \\
\hline Alive & 54 & $(71.1)$ & 22 & $(28.9)$ & 0.006 & 40 & (53.3) & 35 & $(46.7)$ & 0.002 \\
\hline Dead & 11 & $(40.7)$ & 16 & $(59.3)$ & & 4 & $(16.7)$ & 20 & (83.3) & \\
\hline
\end{tabular}

Abbreviations: $p C R$ pathological complete response, $P=P$-value, $S D$ standard deviation, $B M I$ body mass index, ASA American Society of Anesthesiologists, $L A R$ low anterior resection, $A P R$ abdominoperineal excision. Significant $p$-values are represented in bold type 
Table 3 Tumor budding and associations with pathological features

\begin{tabular}{|c|c|c|c|c|c|c|c|c|c|c|}
\hline \multirow{3}{*}{ All patients, n (\%) } & \multicolumn{5}{|c|}{ Budding on H\&E } & \multicolumn{5}{|c|}{ Budding on $\mathrm{IHC}$} \\
\hline & \multicolumn{2}{|c|}{ negative } & \multicolumn{2}{|c|}{ positive } & \multirow[t]{2}{*}{$P$} & \multicolumn{2}{|c|}{ negative } & \multicolumn{2}{|c|}{ positive } & \multirow[t]{2}{*}{$P$} \\
\hline & 65 & $(63.1)$ & 38 & $(36.9)$ & & 44 & $(44.4)$ & 55 & (55.6) & \\
\hline \multicolumn{11}{|l|}{ Tumor size } \\
\hline Mean, cm (SD) & 2.9 & (1.4) & 3.4 & $(2.2)$ & 0.192 & 2.7 & (1.4) & 3.3 & (2.0) & 0.064 \\
\hline \multicolumn{11}{|l|}{ CRM } \\
\hline Mean, mm (SD) & 18.4 & (18.3) & 9.4 & (15.8) & 0.010 & 19.1 & (15.6) & 12.4 & (19.3) & 0.061 \\
\hline \multicolumn{11}{|l|}{ Quirke Grade, n (\%) } \\
\hline Poor & 0 & (0) & 5 & (100) & 0.004 & 0 & (0) & 4 & (100) & 0.218 \\
\hline Suboptimal & 13 & $(81.3)$ & 3 & (18.8) & & 8 & $(50.0)$ & 8 & $(50.0)$ & \\
\hline Optimal & 49 & $(69.0)$ & 22 & $(31.0)$ & & 34 & $(48.6)$ & 36 & (51.4) & \\
\hline Not described & 3 & & 8 & & & 2 & & 7 & & \\
\hline \multicolumn{11}{|l|}{ ypT stage, n (\%) } \\
\hline T0-1 & 10 & (100) & 0 & (0) & 0.001 & 9 & $(90.0)$ & 1 & $(10.0)$ & 0.001 \\
\hline $\mathrm{T} 2$ & 26 & (81.3) & 6 & (18.8) & & 19 & $(61.3)$ & 12 & (38.7) & \\
\hline T3 & 25 & $(49.0)$ & 26 & $(51.0)$ & & 15 & (30.6) & 34 & $(69.4)$ & \\
\hline T4 & 4 & $(40.0)$ & 6 & $(60.0)$ & & 1 & $(11.1)$ & 8 & (88.9) & \\
\hline \multicolumn{11}{|l|}{ ypN stage, n (\%) } \\
\hline No & 40 & (76.9) & 12 & $(23.1)$ & 0.006 & 29 & $(58.0)$ & 21 & $(42.0)$ & 0.021 \\
\hline N1 & 20 & (54.1) & 17 & $(45.9)$ & & 12 & $(32.4)$ & 25 & $(67.6)$ & \\
\hline N2 & 5 & (35.7) & 9 & $(64.3)$ & & 3 & $(25.0)$ & 9 & (75.0) & \\
\hline \multicolumn{11}{|l|}{ Grading, n (\%) } \\
\hline G1 & 2 & $(66.7)$ & 1 & (33.3) & 0.021 & 2 & $(66.7)$ & 1 & (33.3) & 0.290 \\
\hline $\mathrm{G} 2$ & 47 & $(67.1)$ & 23 & $(32.9)$ & & 31 & $(44.9)$ & 38 & (55.1) & \\
\hline G3 & 16 & $(64.0)$ & 9 & $(36.0)$ & & 11 & $(47.8)$ & 12 & (52.2) & \\
\hline G4 & 0 & $(0)$ & 5 & (100) & & 0 & (0) & 4 & (100) & \\
\hline \multicolumn{11}{|c|}{ Dworak's regression, n (\%) } \\
\hline Grade 1 & 2 & $(20.0)$ & 8 & $(80.0)$ & 0.001 & 1 & $(10.0)$ & 9 & $(90.0)$ & 0.001 \\
\hline Grade 2 & 47 & $(62.7)$ & 28 & $(37.3)$ & & 29 & $(40.8)$ & 42 & $(59.2)$ & \\
\hline Grade 3 & 16 & $(88.9)$ & 2 & $(11.1)$ & & 14 & (77.8) & 4 & $(22.2)$ & \\
\hline \multicolumn{11}{|c|}{ Venous invasion, n (\%) } \\
\hline Vo & 58 & $(68.2)$ & 27 & $(31.8)$ & 0.030 & 38 & $(46.3)$ & 44 & $(53.7)$ & 0.404 \\
\hline V1 & 7 & (38.9) & 11 & $(61.1)$ & & 6 & (35.3) & 11 & $(64.7)$ & \\
\hline \multicolumn{11}{|c|}{ Lymphatic invasion, n (\%) } \\
\hline LO & 61 & $(66.3)$ & 31 & $(33.7)$ & 0.055 & 44 & $(50.0)$ & 44 & $(50.0)$ & 0.001 \\
\hline L1 & 4 & (36.4) & 4 & $(63.6)$ & & 0 & (0) & 11 & (100) & \\
\hline \multicolumn{11}{|c|}{ Perineural invasion, n (\%) } \\
\hline Pno & 62 & $(71.3)$ & 25 & $(28.7)$ & 0.001 & 43 & $(51.2)$ & 41 & $(48.8)$ & 0.001 \\
\hline Pn1 & 3 & (18.8) & 13 & $(81.3)$ & & 1 & $(6.7)$ & 14 & $(93.3)$ & \\
\hline \multicolumn{11}{|c|}{ Resection margin, n (\%) } \\
\hline Ro & 64 & $(66.0)$ & 33 & $(34.0)$ & 0.021 & 43 & $(45.7)$ & 51 & $(54.3)$ & 0.792 \\
\hline R1 & 1 & $(20.0)$ & 4 & $(80.0)$ & & 1 & $(25.0)$ & 3 & $(75.0)$ & \\
\hline R2 & 0 & (0) & 1 & $(100)$ & & 0 & (0) & 1 & (100) & \\
\hline
\end{tabular}

Abbreviations: $p C R$ pathological complete response, $P P$-value, $S D$ standard deviation, $C R M$ circumferential resection margin. Significant $p$-values are represented in bold type 
Table 4 Univariate cox regression analysis of DFS

\begin{tabular}{|c|c|c|c|}
\hline \multirow[b]{2}{*}{ Parameter } & \multicolumn{3}{|c|}{ Disease-free survival } \\
\hline & $\mathrm{HR}$ & $95 \% \mathrm{Cl}$ & $P$ \\
\hline Age & 1.03 & {$[0.99 ; 1.06]$} & 0.124 \\
\hline Male vs. female & 0.63 & {$[0.32 ; 1.24]$} & 0.177 \\
\hline BMl & 0.91 & {$[0.85 ; 0.98]$} & 0.009 \\
\hline ASA 2 and 3 & 2.04 & {$[0.62 ; 6.66]$} & 0.238 \\
\hline cM1 & 7.58 & {$[3.42 ; 16.79]$} & $<0.001$ \\
\hline Type of resection: APR vs. LAR & 1.83 & {$[0.92 ; 3.62]$} & 0.083 \\
\hline Adjuvant therapy recieved & 0.99 & {$[0.44 ; 2.21]$} & 0.979 \\
\hline Tumor size & 1.02 & {$[1.00 ; 1.04]$} & 0.048 \\
\hline CRM & 0.93 & {$[0.88 ; 0.97]$} & 0.002 \\
\hline Poor Quirke Grade & 1.78 & {$[0.99 ; 3.18]$} & 0.053 \\
\hline Higher ypT stage [ypT3-4] & 4.20 & {$[1.83 ; 9.66]$} & 0.001 \\
\hline ypN+ & 2.34 & {$[1.16 ; 4.68]$} & 0.017 \\
\hline Histological Grading & 1.97 & {$[1.20 ; 3.25]$} & 0.008 \\
\hline Tumor regression Grading & 0.35 & {$[0.16 ; 0.75]$} & 0.007 \\
\hline Budding positive H\&E & 3.54 & {$[1.82 ; 6.89]$} & $<0.001$ \\
\hline Budding positive $\mathrm{IHC}$ & 6.23 & {$[2.57 ; 15.31]$} & $<0.001$ \\
\hline V1 & 2.41 & {$[1.16 ; 5.03]$} & 0.019 \\
\hline L1 & 2.75 & {$[1.20 ; 6.32]$} & 0.017 \\
\hline $\mathrm{Pn} 1$ & 5.49 & {$[2.65 ; 11.37]$} & 0.001 \\
\hline R+ & 1.51 & {$[0.36 ; 6.35]$} & 0.573 \\
\hline
\end{tabular}

Abbreviations: DFS disease-free survival, $H R$ hazard ratio, 95\% Cl 95\% confidence interval, $P$ P-value, $B M I$ body mass index, ASA American Society of Anesthesiologists, APR abdominoperineal excision, $L A R$ low anterior resection, $C R M$ circumferential resection margin, $P n 1$ perineural invasion; $\mathrm{R}+=$ invaded margin. Significant $p$-values and corresponding hazard ratios are represented in bold type

described in patients with chemotherapy-naïve colorectal cancer [4-9] and included as a recommendation in major national guidelines for the assessment of early invasive cancer $[11,13,14]$.

Budding has been described as a prognostic feature after chemoradiotherapy in rectal cancer patients in several publications with the general limitation of a retrospective study design. In previous studies, budding was reported in 10.1-63.2\% of cases due to different methodologies used for evaluation [3, 15-20]. Budding has been shown to be a negative prognostic factor for survival in different kinds of study designs and for a broad range of cut-offs. However, most of the previous studies could demonstrate effects on survival only in univariate analysis or limited to disease free survival [15-19]. Including patients with complete response in the analysis appeared to attenuate the prognostic impact of tumor budding. In our opinion, it is self-evident that budding cannot be evaluated in patients with a complete response. Therefore, in our study, we focused on cases with poor response in order to stratify the outcome of patients with residual tumor burden. By this approach, we were able
Table 5 Univariate cox regression analysis of OS

\begin{tabular}{|c|c|c|c|}
\hline \multirow[b]{2}{*}{ Parameter } & \multicolumn{3}{|c|}{ Overall survival } \\
\hline & $\mathrm{HR}$ & $95 \% \mathrm{Cl}$ & P \\
\hline Age & 1.06 & {$[1.01 ; 1.10]$} & 0.010 \\
\hline Male vs. female & 0.54 & {$[0.25 ; 1.16]$} & 0.114 \\
\hline BMI & 0.97 & {$[0.84 ; 0 ; 97]$} & 0.006 \\
\hline ASA 2 and 3 & 2.21 & {$[0.52 ; 9.37]$} & 0.282 \\
\hline cM1 & 6.53 & {$[2.74 ; 15.54]$} & 0.001 \\
\hline Type of resection: APR vs. LAR & 1.77 & {$[0.80 ; 3.89]$} & 0.156 \\
\hline Adjuvant therapy & 1.23 & {$[0.45 ; 3.37]$} & 0.681 \\
\hline Tumor size & 1.01 & {$[0.98 ; 1.03]$} & 0.554 \\
\hline CRM & 0.95 & {$[0.90 ; 0.99]$} & 0.043 \\
\hline Poor Quirke Grade & 2.72 & {$[1.47 ; 2.47]$} & 0.001 \\
\hline Higher ypT stage [ypT3-4] & 3.01 & {$[1.20 ; 7.55]$} & 0.019 \\
\hline ypN+ & 1.64 & {$[0.74 ; 3.63]$} & 0.220 \\
\hline Histological Grading & 2.24 & {$[1.26 ; 3.97]$} & 0.006 \\
\hline Tumor regression Grading & 0.19 & {$[0.07 ; 0.51]$} & 0.001 \\
\hline Budding positive H\&E & 3.43 & {$[1.57 ; 7.52]$} & 0.002 \\
\hline Budding positive $\mathrm{IHC}$ & 5.76 & {$[1.95 ; 17.01]$} & 0.002 \\
\hline V1 & 1.60 & {$[0.64 ; 3.99]$} & 0.316 \\
\hline L1 & 1.90 & {$[0.70 ; 5.18]$} & 0.209 \\
\hline Pn1 & 3.76 & {$[1.60 ; 8.85]$} & 0.002 \\
\hline $\mathrm{R}+$ & 1.01 & {$[0.14 ; 7.49]$} & 0.993 \\
\hline
\end{tabular}

Abbreviations: OS overall survival, $H R$ hazard ratio, $95 \% \mathrm{Cl} 95 \%$ confidence interval, P P-value, BMI body mass index, ASA American Society of Anesthesiologists, APR abdominoperineal excision, $L A R$ low anterior resection, $C R M$ circumferential resection margin, $P n 1$ perineural invasion, $\mathrm{R}+=$ invaded margin. Significant $p$-values and corresponding hazard ratios are represented in bold type

to demonstrate a strong impact on disease free survival and overall survival in univariate and multivariate analysis.

Of the most recent studies, Jäger et al. [3] can be compared to our own study. As in our study, they evaluated budding not only at the invasive front but also throughout the tumor. The high budding rate of $63.2 \%$ compared to our results can be explained by the low cut-off of two buds in one microscopic field, whereas in our study a cutoff of 5 buds was used according to standard criteria of Ueno et al. [10]. As in our study, budding remained a significant parameter in multivariate analysis

Table 6 Multivariate cox regression analysis of DFS: Budding evaluated on H\&E

\begin{tabular}{llll}
\hline \multirow{2}{*}{ Parameter } & \multicolumn{3}{l}{ Disease-free survival } \\
\cline { 2 - 4 } & $\mathrm{HR}$ & $95 \% \mathrm{Cl}$ & $\mathrm{P}$ \\
\hline Positive Budding H\&E & $\mathbf{2 . 3 4}$ & {$[1.14 ; 4.79]$} & $\mathbf{0 . 0 2 0}$ \\
Higher ypT stage [ypT3-4] & $\mathbf{2 . 8 5}$ & {$[1.16 ; 7.02]$} & $\mathbf{0 . 0 2 3}$ \\
ypN + & 1.34 & {$[0.63 ; 2.83]$} & 0.449 \\
\hline
\end{tabular}


Table 7 Multivariate cox regression analysis of DFS: Budding evaluated on $\mathrm{IHC}$

\begin{tabular}{llll}
\hline \multirow{2}{*}{ Parameter } & \multicolumn{3}{l}{ Disease-free survival } \\
\cline { 2 - 4 } & $\mathrm{HR}$ & $95 \% \mathrm{Cl}$ & $\mathrm{P}$ \\
\hline Positive Budding IHC & $\mathbf{4 . 5 9}$ & {$[1.79 ; 11.72]$} & $\mathbf{0 . 0 0 1}$ \\
Higher ypT stage [ypT3-4] & 2.16 & {$[0.87 ; 5.34]$} & 0.095 \\
ypN+ & 1.29 & {$[0.60 ; 2.77]$} & 0.516 \\
\hline
\end{tabular}

for disease free survival. However they failed to demonstrate this for overall survival, presumably, because patients with complete response were included in the statistical analysis.

Only one previous study claimed that a single cell pattern of growth in the invasive front was a prognostic factor for prolonged cancer-specific survival [21]. They interpreted the single-cell growth pattern as an indicator of tumor cell regression. However, they did not evaluate budding as a standardized parameter but rather as a semiquantitative score of the tumor growth pattern. Furthermore, patients with a complete pathological response were included in the survival analysis, undermining the impact of budding as a parameter for the stratification of patients with a poor response.

In our study, immunohistochemical staining showed that budding had a considerable prognostic influence and was even superior to that of conventional parameters such as ypT and ypN stage, which have been used in routine so far. Therefore, the assumption arises that the assessment of posttreatment budding may improve the commonly used TNM classification for stratifying rectal cancer patients treated with neoadjuvant therapy and for predicting prognosis.

However, there is still a general lack of a unified definition of tumor budding. At the International Tumor Budding Consensus Conference (ITBCC) in 2016, a consensus for a standardized definition of budding and for an evaluation method was reached, but only for colorectal cancers without neoadjuvant chemoradiotherapy so far [22]. Tumor budding was defined as a single cell or a cluster of up to four tumor cells assessed in one hotspot measuring $0.785 \mathrm{~mm}^{2}$ at the invasive front. Furthermore, a three-tier system was recommended with a whole budding count to allow adequate risk stratification. The documentation of tumor budding after neoadjuvant

Table 8 Multivariate cox regression analysis of OS: Budding evaluated on H\&E

\begin{tabular}{llll}
\hline \multirow{2}{*}{ Parameter } & \multicolumn{3}{l}{ Overall survival } \\
\cline { 2 - 4 } & $\mathrm{HR}$ & $95 \% \mathrm{Cl}$ & $P$ \\
\hline Positive Budding H\&E & $\mathbf{2 . 7 2}$ & {$[1.15 ; 6.44]$} & $\mathbf{0 . 0 2 3}$ \\
Higher ypT stage [ypT3-4] & 2.17 & {$[0.78 ; 6.06]$} & 0.140 \\
ypN + & 1.12 & {$[0.46 ; 2.71]$} & 0.803 \\
\hline
\end{tabular}

Table 9 Multivariate cox regression analysis of OS: Budding evaluated on $\mathrm{IHC}$

\begin{tabular}{llll}
\hline \multirow{2}{*}{ Parameter } & \multicolumn{4}{l}{ Overall survival } \\
\cline { 2 - 4 } & $\mathrm{HR}$ & $95 \% \mathrm{Cl}$ & $\boldsymbol{P}$ \\
\hline Positive Budding IHC & $\mathbf{5 . 1 9}$ & {$[1.62 ; 16.61]$} & $\mathbf{0 . 0 0 6}$ \\
Higher ypT stage [ypT3-4] & 1.50 & {$[0.53 ; 4.26]$} & 0.443 \\
ypN+ & 1.18 & {$[0.47 ; 2.92]$} & 0.727 \\
\hline $\begin{array}{l}\text { Abbreviations: DFS disease-free survival, OS overall survival, HR hazard ratio, } \\
\text { 95\% Cl 95\% confidence interval, } P \text { P-value. Significant } p \text {-values and } \\
\text { corresponding hazard ratios are represented in bold type }\end{array}$
\end{tabular}

therapy has not yet been suggested for daily diagnostic practice because of data gaps in its prognostic value in treated rectal cancers as well as a lack of a standardized evaluation method for these cancers.

The initial morphology of the tumor is often modified after chemoradiation, with phenomena such as heavy fibrosis, breaking up of the glandular tumor structures and necrotic areas. Due to these factors, the assessment of budding proposed by the ITBCC becomes challenging. It should also be mentioned that after neoadjuvant therapy, tumor borders may appear fragmented, so the tumors occasionally form several invasive fronts in the context of fibrosis and inflammation. Owing to these histological changes, we assessed tumor budding not only at the utmost invasive front (such as in cancers without chemoradiation) but also in-between invasive foci. Lugli et al. [23] and Rieger et al. [24] showed that intratumoral budding in chemotherapy-naïve patients with colorectal cancer is generally associated with peritumoral budding. They found that as long as the observer investigates the densest region with budding, it does not matter whether buds are detected at the invasive front or within the tumor. Our method used to assess budding without being limited to the invasive front in neoadjuvant-treated cancers was fundamentally based on those findings. With our method, we were able to address the abovementioned problems occurring after preoperative therapy while keeping the method relatively simple and potentially reproducible for other observers.

For our budding analyses, we used the one hotspot method, as recommended by the ITBCC and originally proposed by Ueno et al. [10]. It is a fast and simple way to subdivide patients into two different categories that are prognostically highly relevant. Even in patients with little residual tumor after preoperative therapy, the method was able to find high-risk patients. Although the cut-off was set to merely 5 buds per hotspot, as proposed for the stratification of pT1 carcinomas in polyps [10], it was possible to apply the same cut-off for locally advanced cancers, with a resulting high impact of both disease-free survival and overall survival.

In addition to $\mathrm{H} \& \mathrm{E}$ staining, we used IHC staining to make buds more readily visible. Kai et al. [25] were able 

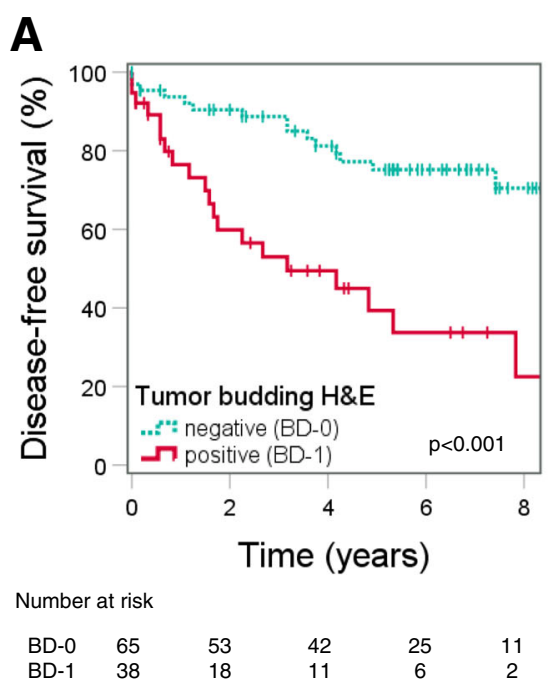

C

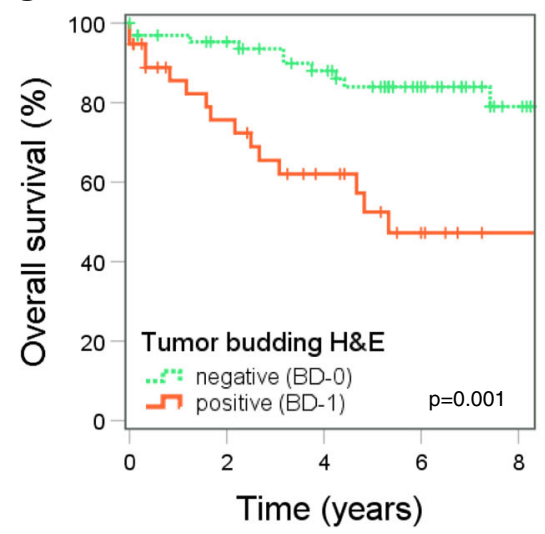

Number at risk

$\begin{array}{llllcc}\text { BD-0 } & 65 & 56 & 46 & 27 & 12 \\ \text { BD-1 } & 38 & 23 & 15 & 8 & 3\end{array}$

B

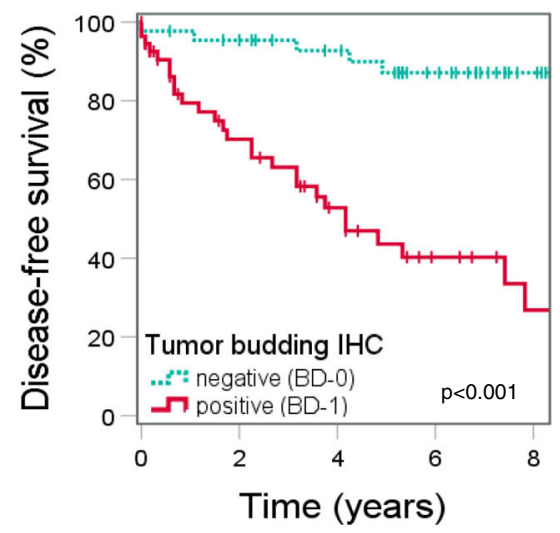

Number at risk

D

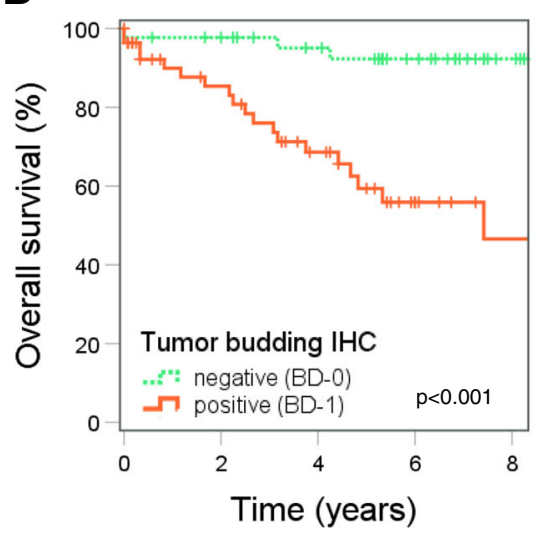

Number at risk

$\begin{array}{llllll}\text { BD-0 } & 44 & 41 & 35 & 23 & 10 \\ \text { BD-1 } & 55 & 37 & 25 & 12 & 5\end{array}$

Fig. 3 Kaplan-Meier curves for disease-free survival (DFS) and overall survival (OS). Independently of the staining method, DFS and OS were significant poorer on budding positive cases (BD-1). a DFS and budding evaluated on H\&E (Log-rank test $p<0.001$ ). $\mathbf{b}$ DFS and budding evaluated on IHC (Log-rank test $p<0.001)$. c OS and budding evaluated on H\&E (Log-rank test $p=0.001$ ). $\mathbf{d}$ OS and budding evaluated on IHC (Log-rank test $p<0.001)$

to show that IHC can reduce interobserver variability in the evaluation of budding between unskilled observers. This would make IHC suitable for training pathologists who are inexperienced in this field. As described in previous studies for colorectal cancers without prior chemotherapy, cytokeratin staining detected more budding-positive cases [26]. In our study, we detected more budding-positive cases by the means of IHC staining as well, and this method improved the prognostic value of tumor budding assessment. IHC helped to stratify patients into even more meaningful risk groups than H\&E staining. When analyzed with IHC staining, fewer budding-negative cases were found, and these had a better prognosis than budding-negative cases found by $H \& E$ (five-year disease-free survival rate: $87 \%$ vs $75 \%$; and five-year overall survival rate: $92 \%$ vs $84 \%$ ). The employed cut-off may therefore identify patients with a favorable prognosis who might be able to refrain from adjuvant therapy. If the cut-off for budding on IHC was higher, high-risk patients would more likely come to light. In these patients, more intensive aftercare might be recommended. The ideal cut-off for the evaluation of budding in IHC-stained sections still needs to be investigated. In tumors without neoadjuvant therapy, the ITBCC recommends the use of IHC in difficult cases (such as for distinguishing buds from peritumoral inflammation reaction), but the final evaluation should still be performed on H\&E-stained slides [22]. In the case of pretreated tumors, the role of IHC and H\&E staining still needs to be determined. Since, for example, more 
tumor inflammation occurs in such cases, IHC might play a more central role in the evaluation of these tumors. So far, only two previous studies regarding IHC staining in posttreatment budding exist $[17,18]$. Concerning this issue, more investigations should be performed.

The main limitation of this work is its retrospective nature. It also needs to be determined whether the number and composition of included patients may vary among patients with different ethnic backgrounds. In addition, the recorded number of events during the follow-up could be a limitation as well, especially in the interpretation of the Cox regression models due to the possible lack of outcome data. Nevertheless, current data on the prognosis of tumor budding in rectal cancers treated with neoadjuvant therapy are unsatisfactory.

It is remarkable that lymph node status did not have a significant effect on outcome in our multivariate survival analysis. In fact, previous studies of neoadjuvant therapy in rectal cancer found strong associations of lymph node status with survival $[27,28]$. However, in these studies, patients with complete pathological response were included in the analysis, who were here excluded due to being non-informative for tumor budding. We speculate that the specific case selection in our study may be the cause of such discrepancies.

\section{Conclusion}

In conclusion, our study provides further information to understand and evaluate budding in rectal carcinomas treated with neoadjuvant therapy while analyzing budding on H\&E- and immunostained specimens. Immunohistochemical staining can substantially enhance the diagnostic accuracy and prognostic impact. Tumor budding should be taken more seriously into account in daily diagnostic practice, since it represents an additional and independent prognostic factor for therapeutic decision making, even in rectal cancer patients after preoperative therapy. The method of Ueno is well suited for this purpose because of its fast and simple method of evaluating budding-positive cases. However, more knowledge about budding and a consensus about the best method used to assess buds after perioperative therapy are strongly needed to standardize the process and to speed up its application in pathological protocols for treated rectal cancers.

\section{Abbreviations}

5-FU: 5-fluorouracil; 95\% Cl: 95\% confidence interval; APR: Abdominoperineal excision; ASA: American Society of Anesthesiologists; BD-0: Budding negative; BD-1: Budding positive; BMI: Body mass index; CRM: Circumferential resection margin;; CT: Computed tomography; DAB: 3,3'-diaminobenzidintetrahydrochlorid; DFS: Disease-free survival; Fig: Figure; Gy: Grey; H\&E: hematoxylin-eosin staining; HR: Hazard ratio; HRP: Horseradishperoxidase; IHC: Immunohistochemical staining; LAR: Low anterior resection; MeV: electronvolt; OS: Overall survival; P: P-value; pCR: pathological complete response; SD: Standard deviation; TME: Total mesorectal surgery;

TNM: Tumor, node, and metastasis staging system

\section{Acknowledgements}

We thank Ines Koch for expert technical assistance.

\section{Authors' contributions}

IT and SE developed methodology and performed experiments; IT, HS, AJM, $\mathrm{KN}, \mathrm{MK}, \mathrm{DH}$ and SE analyzed and interpreted data, and wrote the

manuscript; SE supervised the study. All authors read and approved the final manuscript.

\section{Funding}

There are no funding sources.

Availability of data and materials

The datasets used and/or analyzed during the current study are available from the corresponding author on reasonable request.

\section{Ethics approval and consent to participate}

Investigations on archived tissue and anonymized data were approved by the Charité Ethics Committee (Ethikkomission der Charité -

Universitätsmedizin Berlin), decision №. EA1/370/16.

All patients provided written consent to further investigate their tissue samples as well as the anonymous use of their clinical data.

Consent for publication

Not applicable.

\section{Competing interests}

The authors declare that they have no competing interests.

\section{Author details}

${ }^{1}$ Institut für Pathologie, Charité - Universitätsmedizin Berlin, Campus Charité Mitte, Charitéplatz 1, 10117 Berlin, Germany. ${ }^{2}$ Institut für Biometrie und Klinische Epidemiologie, Charité - Universitätsmedizin Berlin, Charitéplatz 1 , 10117 Berlin, Germany. ${ }^{3}$ Berlin Institute of Health (BIH), Anna-Louisa-Karsch 2, 10178 Berlin, Germany. ${ }^{4}$ Klinik für Allgemein- und Viszeralchirurgie Klinikum, Müllroser Chaussee 7, 15236 Frankfurt (Oder), Germany.

Received: 4 July 2019 Accepted: 15 October 2019

Published online: 01 November 2019

References

1. Sauer R, Liersch T, Merkel S, Fietkau R, Hohenberger W, Hess C, Becker H, Raab HR, Villanueva MT, Witzigmann $H$, et al. Preoperative versus postoperative chemoradiotherapy for locally advanced rectal cancer: results of the German CAO/ARO/AIO-94 randomized phase III trial after a median follow-up of 11 years. J Clin Oncol. 2012;30(16):1926-33.

2. Sauer $R$, Becker $H$, Hohenberger W, Rödel C, Wittekind C, Fietkau R, Martus $P$, Tschmelitsch J, Hager E, Hess CF, et al. Preoperative versus postoperative chemoradiotherapy for rectal cancer. N Engl J Med. 2004;351(17):1731-40.

3. Jäger $T$, Neureiter $D$, Fallaha $M$, Schredl $P$, Kiesslich $T$, Urbas R, Klieser $E$, Holzinger J, Sedlmayer F, Emmanuel K, et al. The potential predictive value of tumor budding for neoadjuvant chemoradiotherapy response in locally advanced rectal cancer. Strahlenther Onkol. 2018;194(11):991-1006.

4. Hase K, Shatney C, Johnson D, Trollope M, Vierra M. Prognostic value of tumor "budding" in patients with colorectal cancer. Dis Colon Rectum. 1993; 36(7):627-35.

5. Wang LM, Kevans D, Mulcahy H, O'Sullivan J, Fennelly D, Hyland J, O'Donoghue D, Sheahan K. Tumor budding is a strong and reproducible prognostic marker in T3N0 colorectal cancer. Am J Surg Pathol. 2009;33(1): 134-41.

6. Okuyama T, Oya M, Ishikawa H. Budding as a useful prognostic marker in PT3 well- or moderately-differentiated rectal adenocarcinoma. J Surg Oncol. 2003:83(1):42-7.

7. Ueno H, Murphy J, Jass JR, Mochizuki H, Talbot IC. Tumour 'budding' as an index to estimate the potential of aggressiveness in rectal cancer. Histopathology. 2002;40(2):127-32. 
8. Kanazawa H, Mitomi H, Nishiyama Y, Kishimoto I, Fukui N, Nakamura T, Watanabe M. Tumour budding at invasive margins and outcome in colorectal cancer. Color Dis. 2008;10(1):41-7.

9. Nakamura T, Mitomi H, Kikuchi S, Ohtani Y, Sato K. Evaluation of the usefulness of tumor budding on the prediction of metastasis to the lung and liver after curative excision of colorectal cancer. Hepatogastroenterology. 2005;52(65):1432-5.

10. Ueno H, Mochizuki H, Hashiguchi Y, Shimazaki H, Aida S, Hase K, Matsukuma S, Kanai T, Kurihara H, Ozawa K, et al. Risk factors for an adverse outcome in early invasive colorectal carcinoma. Gastroenterology. 2004; 127(2):385-94.

11. Leitlinienprogramm Onkologie (Deutsche Krebsgesellschaft DK, AWMF): S3Leitlinie Kolorektales Karzinom, Langversion 2.1, 2019, AWMF Registrierungsnummer: 021/0070L, http://www.leitlinienprogrammonkologie.de/leitlinien/kolorektales-karzinom/. Accessed 13 Feb 2019.

12. Dworak O, Keilholz L, Hoffmann A. Pathological features of rectal cancer after preoperative radiochemotherapy. Int J Color Dis. 1997;12(1):19-23.

13. Watanabe T, Muro K, Ajioka Y, Hashiguchi Y, Ito Y, Saito Y, Hamaguchi T, Ishida H, Ishiguro M, Ishihara S, et al. Japanese Society for Cancer of the Colon and Rectum (JSCCR) guidelines 2016 for the treatment of colorectal cancer. Int J Clin Oncol. 2017.

14. Kakar S SC, Berho ME, Driman DK, Fitzgibbons P, Frankel WL, hill KA, Jessup J, Krasinskas AM, Washington MK: Protocol for the Examination of Specimens From Patients With Primary Carcinoma of the Colon and Rectum. 2017. https://documents.cap.org/protocols/cp-gilowercolonrectum-17protocol-4010.pdf. Accessed 12 Sep 2019.

15. Huebner M, Wolff BG, Smyrk TC, Aakre J, Larson DW. Partial pathologic response and nodal status as most significant prognostic factors for advanced rectal cancer treated with preoperative chemoradiotherapy. World J Surg. 2012;36(3):675-83.

16. Sannier A, Lefèvre JH, Panis Y, Cazals-Hatem D, Bedossa P, Guedj N. Pathological prognostic factors in locally advanced rectal carcinoma after neoadjuvant radiochemotherapy: analysis of 113 cases. Histopathology. 2014;65(5):623-30

17. Du C, Xue W, Li J, Cai Y, Gu J. Morphology and prognostic value of tumor budding in rectal cancer after neoadjuvant radiotherapy. Hum Pathol. 2012; 43(7):1061-7.

18. Bhangu A, Wood G, Brown G, Darzi A, Tekkis P, Goldin R. The role of epithelial mesenchymal transition and resistance to neoadjuvant therapy in locally advanced rectal cancer. Color Dis. 2014;16(4):0133-43.

19. Demir A, Alan O, Oruc E. Tumor budding for predicting prognosis of resected rectum cancer after neoadjuvant treatment. World J Surg Oncol. 2019;17(1):50

20. Şirin AH, Sökmen S, Ünlü SM, Ellidokuz H, Sarioğlu S. The prognostic value of tumor budding in patients who had surgery for rectal cancer with and without neoadjuvant therapy. Tech Coloproctol. 2019;23(4):333-42.

21. Jessberger J, Erlenbach-Wünsch $K$, Posselt $R$, Haderlein M, Agaimy A, Fietkau R, Hartmann A, Distel L. Combination of growth pattern and tumor regression identifies a high-risk group in neoadjuvant treated rectal cancer patients. J Dig Dis. 2017;18(5):283-91.

22. Lugli A, Kirsch R, Ajioka Y, Bosman F, Cathomas G, Dawson H, El Zimaity H, Fléjou JF, Hansen TP, Hartmann A, et al. Recommendations for reporting tumor budding in colorectal cancer based on the international tumor budding consensus conference (ITBCC) 2016. Mod Pathol. 2017;30(9):1299-311.

23. Lugli A, Vlajnic T, Giger O, Karamitopoulou E, Patsouris ES, Peros G, Terracciano LM, Zlobec I. Intratumoral budding as a potential parameter of tumor progression in mismatch repair-proficient and mismatch repairdeficient colorectal cancer patients. Hum Pathol. 2011;42(12):1833-40.

24. Rieger $\mathrm{G}$, Koelzer $\mathrm{VH}$, Dawson $\mathrm{HE}$, Berger MD, Hadrich M, Inderbitzin D, Lugli A, Zlobec I. Comprehensive assessment of tumour budding by cytokeratin staining in colorectal cancer. Histopathology. 2017;70(7):1044-51.

25. Kai K, Aishima S, Aoki S, Takase Y, Uchihashi K, Masuda M, NishijimaMatsunobu A, Yamamoto M, Ide K, Nakayama A, et al. Cytokeratin immunohistochemistry improves interobserver variability between unskilled pathologists in the evaluation of tumor budding in $\mathrm{T} 1$ colorectal cancer Pathol Int. 2016;66(2):75-82.

26. Mitrovic B, Schaeffer DF, Riddell RH, Kirsch R. Tumor budding in colorectal carcinoma: time to take notice. Mod Pathol. 2012;25(10):1315-25.

27. Fokas E, Liersch T, Fietkau R, Hohenberger W, Beissbarth T, Hess C, Becker H, Ghadimi M, Mrak K, Merkel S, et al. Tumor regression grading after preoperative Chemoradiotherapy for locally advanced rectal carcinoma revisited: updated results of the CAO/ARO/AIO-94 trial. J Clin Oncol. 2014; 32(15):1554-62

28. Rödel C, Martus P, Papadoupolos T, Füzesi L, Klimpfinger M, Fietkau R, Liersch T, Hohenberger W, Raab R, Sauer R, et al. Prognostic significance of tumor regression after preoperative chemoradiotherapy for rectal cancer. J Clin Oncol. 2005;23(34):8688-96.

\section{Publisher's Note}

Springer Nature remains neutral with regard to jurisdictional claims in published maps and institutional affiliations.

\section{Ready to submit your research? Choose BMC and benefit from:}

- fast, convenient online submission

- thorough peer review by experienced researchers in your field

- rapid publication on acceptance

- support for research data, including large and complex data types

- gold Open Access which fosters wider collaboration and increased citations

- maximum visibility for your research: over $100 \mathrm{M}$ website views per year

At BMC, research is always in progress.

Learn more biomedcentral.com/submissions 